\title{
Quercetin-mediated apoptosis via activation of the mitochondrial- dependent pathway in MG-63 osteosarcoma cells
}

\author{
WENNA LIANG ${ }^{1}$, XIHAI LI ${ }^{2}$, CANDONG LI $^{1}$, LIANMING LIAO ${ }^{2}$, BIZHENG GAO $^{1}$, HUIJUAN GAN ${ }^{1}$, \\ ZHAOYANG YANG ${ }^{1}$, LINGHONG LIAO ${ }^{1}$ and XUZHENG CHEN ${ }^{2}$ \\ ${ }^{1}$ Research Base of TCM Syndrome, ${ }^{2}$ Academy of Integrative Medicine, \\ Fujian University of Traditional Chinese Medicine, Fuzhou, Fujian 350108, P.R. China
}

Received February 7, 2011; Accepted July 6, 2011

DOI: $10.3892 / \mathrm{mmr} .2011 .533$

\begin{abstract}
Quercetin (a natural polyphenolic compound) is a polyphenolic flavonoid compound found in a variety of plants. It has been demonstrated to exert cytostatic activity against a variety of human cancer cell lines, including the human osteosarcoma cell line, MG-63. However, its effects on osteosarcoma cell apoptosis are still undefined. The present study was undertaken to examine the effect of quercetin on cell viability, apoptosis and mitochondrial membrane potential, and to determine the molecular mechanism of quercetininduced apoptosis by investigating the expression of $\mathrm{Bcl}-2$ family proteins (Bcl-2, Bax), cytochrome $\mathrm{C}$, caspase-9 and caspase- 3 in MG-63 cells. We found that quercetin suppressed the viability of MG-63 cells in a dose- and time- dependent manner. Furthermore, we observed that quercetin induced the loss of mitochondrial membrane potential, upregulated the expression of the proapoptotic proteins, Bax and cytochrome $\mathrm{C}$, and activated caspase- 9 and caspase- 3 , and downregulated the expression of antiapoptotic protein, $\mathrm{Bcl}-2$. These data suggest that quercetin may induce apoptosis via the mitochondrial-dependent pathway in MG-63 cells.
\end{abstract}

\section{Introduction}

Osteosarcoma (OS) is the third most common cancer in childhood and adolescents, and the most common primary malignant bone tumor (1). With the development of new chemotherapy protocols, surgical techniques and radiological staging, the 5 -year survival and cure rates have increased to $60-80 \%$ in patients with localized disease (2). However, major problems associated with chemotherapy still remain, particularly the frequent development of drug resistance (3). Moreover, chemo-

Correspondence to: Dr CanDong Li, Research Base of TCM Syndrome, Fujian University of Traditional Chinese Medicine, 1 Huatuo Road, University Town, Minhou Shangjie, Fuzhou, Fujian 350108, P.R. China

E-mail: fjzylcd@126.com

Key words: osteosarcoma, apoptosis, quercetin, mitochondria therapy may induce DNA mutations in normal cells. Hence the challenge of seeking new therapeutic approaches that can further improve the efficiency and alleviate the adverse effects is clear and urgent (4). Epidemiological studies have shown that regular consumption of tea, vegetables and fruits is associated with reduced risk of cancer (5). Quercetin with a molecular formula of $\mathrm{C}_{15} \mathrm{H}_{10} \mathrm{O}_{7}$ (Fig. 1), is an abundant flavonoid in tea, fruits and vegetables (6). Quercetin has been shown to induce cell death by apoptosis in many human cancer cell lines, such as hepatoma, oral, leukemia, lung and colon cancer cell lines $(7,8)$.

Apoptotic cell death is an innate cellular response to eliminate abnormal or redundant cells in mammals and hence is crucial for mammal development and tissue homeostasis (9). The disturbed regulation of this vital process represents a major causative factor in the pathogenesis of cancers, including OS $(10,11)$. The mitochondrial-dependent pathway is one of the apoptotic pathways in mammalian cells. Mitochondrial membrane permeabilization, accompanied by the collapse of electrochemical gradient across the mitochondrial membrane causes the release of catabolic hydrolases and activators of such enzymes (including those of caspases) from the mitochondria, leading to cell apoptosis $(12,13)$. Bcl-2 family proteins serve as critical regulators of this pathway through their influence on mitochondrial outer membrane permeabilization (MOMP) following homo- or hetero-association (14). Among Bcl-2 family proteins, pro-apoptotic members, such as Bax and Bak increase MOMP during apoptosis, releasing apoptogenic proteins, such as cytochrome $\mathrm{C}$ and Diablo/Smac that trigger apoptosis by activating caspases and nucleases; whereas antiapoptotic members, such as Bcl-2 may bind to activate Bax to decrease membrane permeability (15). The regulation of active anti- and pro-apoptotic Bcl-2 family members is a key for determining the fate of cells, and disturbance of the normal apoptotic program due to alteration of the ratio by aberrant expression of these proteins may lead to various apoptosisrelated diseases $(16,17)$. In addition, Bcl-2 overexpression, widely found in a variety of cancers, suppresses apoptosis and confers resistance to anticancer drugs. Therefore, the induction of apoptosis through the mitochondrial-dependent pathway has been one of the goals of anti-cancer chemotherapy.

In this study, we evaluated the effect of quercetin on the apoptosis of the human osteosarcoma cell line, MG-63, and investigated the possible underlying molecular mechanisms. 
We found that the quercetin-induced apoptosis of MG-63 cells was accompanied by the loss of mitochondrial membrane potential $(\Delta \psi \mathrm{m})$, downregulation of Bcl-2, upregulation of Bax and cytochrome $\mathrm{C}$, and caspase- 9 and caspase- 3 activation. Our data suggest that quercetin can induce MG-63 cell apoptosis via activation of the mitochondrial-dependent pathway.

\section{Materials and methods}

Materials and reagents. Dulbecco's modified Eagle's medium (DMEM), fetal bovine serum (FBS), trypsin-EDTA, 5,5',6,6'tetrachloro-1,1',3,3'-tetraethyl-benzimidazol-carbocyanine iodide (JC-1) and TRIzol reagent, caspase-9 and caspase-3 colorimetric protease assay kits were provided by Invitrogen (Grand Island, NY, USA). SuperScript II reverse transcriptase was obtained from Promega (Madison, WI, USA). Antibodies against Bcl-2, Bax, cytochrome $\mathrm{C}$ and $\beta$-actin, and horseradish peroxidase (HRP)-conjugated secondary antibodies were purchased from Cell Signaling (Beverly, MA, USA). The fluorescein isothiocyanate (FITC)-conjugated Annexin V apoptosis detection kit was provided by Becton-Dickinson (San Jose, CA, USA). Quercetin was obtained from Sigma Chemical Co. (St. Louis, MO, USA). Stock solutions of quercetin were prepared by dissolving the quercetin powder in DMSO to a concentration of $1 \mathrm{M}$, and stored at $-20^{\circ} \mathrm{C}$. The working concentrations of quercetin were made by diluting the stock solution with the culture medium. The final concentration of DMSO in the medium was $<0.5 \%$.

Cell culture. Human osteosarcoma cell line MG-63 cells from the American Type Culture Collection (Manassas, VA) were maintained at $37^{\circ} \mathrm{C}$ in an atmosphere with $5 \% \mathrm{CO}_{2}$ with DMEM, supplemented with $10 \%$ (v/v) FBS, $100 \mathrm{U} / \mathrm{ml}$ penicillin, and $100 \mu \mathrm{g} / \mathrm{ml}$ streptomycin. MG-63 cells were subcultured at $80-90 \%$ confluency. The cells used in this study were subjected to no more than 20 cell passages.

Cell viability by MTT assay. The cells were cultured in 96-well plates at a concentration of $1 \times 10^{4}$ cells/well. Cell viability was assessed by the 3-(4, 5-dimethylthiazol-2-yl)-2, 5-diphenyltetrazolium bromide (MTT) colorimetric assay. The cells were treated with quercetin at various final concentrations $(20,40$, $80,160,240$ and $320 \mu \mathrm{M}$, respectively), and the vehicle control cells treated with $0.5 \%$ DMSO for $48 \mathrm{~h}$. In some experiments, cells were treated with $160 \mu \mathrm{M}$ of quercetin for different periods of time. After treatment, $10 \mu 1 \mathrm{MTT}$ [5 $\mathrm{mg} / \mathrm{ml}$ in phosphate buffered saline (PBS)] were added to each well, and the samples were incubated at $37^{\circ} \mathrm{C}$ for $4 \mathrm{~h}$. The purple-blue MTT formazan precipitate was dissolved in $100 \mu 1 \mathrm{DMSO}$ and cells were shaken for $10 \mathrm{~min}$. The absorbance was measured at $490 \mathrm{~nm}$ using an ELISA reader (BioTek, Model EXL800, USA).

Observation of morphological changes. MG-63 cells were cultured in 6-well plates at a concentration of $2 \times 10^{5}$ cells/well. The cells were treated with various concentrations of quercetin for 48 h. MG-63 cell morphology was observed using a phasecontrast microscope (Olympus, Japan). The photographs of MG-63 cells were taken at a magnification of x100.

Measurement of apoptosis by flow cytometry analysis with Annexin V/propidium iodide (PI) staining. After treatment with

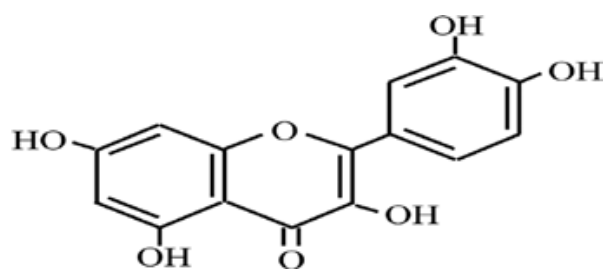

Figure 1. The chemical structure of quercetin.

various concentrations of quercetin, the apoptosis of MG-63 cells was determined by flow cytometry analysis using a fluorescence-activated cell sorting (FACS)calibur (Becton-Dickinson, CA, USA) with Annexin V-fluorescein isothiocyanate (FITC)/ PI staining. The staining was performed according to the manufacturer's instructions. Early apoptotic cells were determined by Annexin V-positivity and PI-negativity, while late apoptotic cells were determined by Annexin V-positivity and PI-positivity.

Detection of $\Delta \psi m$ by flow cytometry analysis with JC-1 staining. JC-1 is a cationic dye that can be used as an indicator of mitochondrial potential. It exhibits mitochondrial potential-dependent accumulation, which can be detected by a fluorescence emission shift from green to red. After treatment with various concentrations of quercetin, MG-63 cells were trypsinized, resuspended in $1 \mathrm{ml}$ of medium and incubated with $10 \mu \mathrm{g} / \mathrm{ml}$ of JC-1 (Invitrogen) at $37^{\circ} \mathrm{C}$ for $30 \mathrm{~min}$. After JC-1 staining, red and green fluorescence emissions were analyzed by flow cytometry.

RNA extraction and RT-PCR analysis. MG-63 cells were cultured in 6-well plates at a concentration of $2 \times 10^{5}$ cells/ well and treated with various concentrations of quercetin for 48 h. Total RNA from MG-63 cells was isolated with TRIzol reagent (Invitrogen). Oligo(dT)-primed RNA (5 $\mu \mathrm{g})$ was reverse transcribed with SuperScript II reverse transcriptase (Promega) according to the manufacturer's instructions. The obtained cDNA was used to determine the mRNA amount of Bcl-2 and Bax by PCR with Taq DNA polymerase (Fermentas). $\beta$-actin was used as the internal control. The primers used for the amplification of $\mathrm{Bcl}-2$, Bax and $\beta$-actin transcripts were as follows: Bcl-2 (310 bp) forward, 5'-CGA CTT CGC CGA GAT GTC CAG CCA G-3' and reverse, 5'-ACT TGT GGC CCA GAT AGG CAC CCA G-3'; Bax (289 bp) forward, 5'-CTG ACA TGT TTT CTG ACG GC-3' and reverse, 5'-TCA GCC CAT CTT CTT CCA GA-3'; $\beta$-actin (285 bp) forward, 5'-AGC GAG CAT CCC CCA AAG TT-3' and reverse, 5'-GGG CAC GAA GGC TCA TCA TT-3'.

Western blot analysis. MG-63 cells were cultured in 6-well plates at a concentration of $2 \times 10^{5}$ cells/well. After treatment with quercetin at various concentrations for $48 \mathrm{~h}$, cells were lysed with mammalian cell lysis buffer (M-PER; Thermo Scientific, Rockford, IL, USA) containing protease and phosphatase inhibitor cocktails (EMD Biosciences and Sigma Chemical, USA, respectively), and protein concentrations were determined by the BCA assay using bovine serum albumin as the standard. The samples (20 $\mu \mathrm{g}$ for each) were loaded and separated by electrophoresis on $12 \%$ SDS-polyacrylamide gels 
A

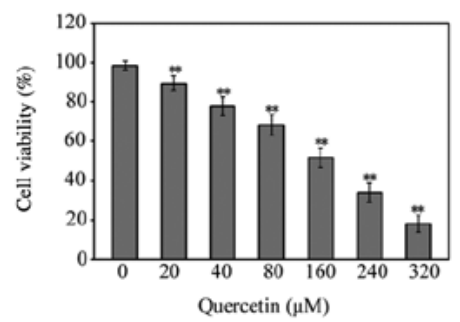

B

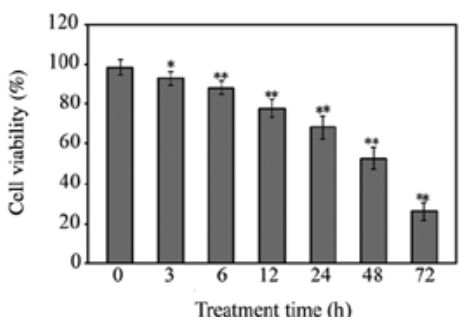

Figure 2. Effect of quercetin on cell viability of MG-63 cells. (A) MG-63 cells were treated with the indicated concentrations of quercetin for $48 \mathrm{~h}$. (B) Cells were treated with $160 \mu \mathrm{M}$ of quercetin for the indicated time-periods. Cell viability was determined by the MTT assay. The data were normalized to the viability of the control cells $(100 \%)$. Data are averages \pm SD (error bars), ${ }^{*} \mathrm{P}<0.05,{ }^{* *} \mathrm{P}<0.01$, compared to the control cells.

under a reducing condition using $200 \mathrm{~V}$ for $1 \mathrm{~h}$. After electrophoresis, the proteins were transferred to PVDF membranes in a Tris-glycine transfer buffer ( $48 \mathrm{mM}$ Tris, $39 \mathrm{mM}$ glycine, $0.05 \% \mathrm{w} / \mathrm{v}$ SDS, $10 \% \mathrm{v} / \mathrm{v}$ methanol) using a semi-dry blotting system, and incubated with antibodies against Bcl-2, Bax, cytochrome $\mathrm{C}$ and $\beta$-actin $(1: 1000)$ overnight at $4^{\circ} \mathrm{C}$ with rocking. After PVDF membranes were washed in TBST, secondary HRP-conjugated antibodies were added at 1:2000 dilution for $1 \mathrm{~h}$ at room temperature and the PVDF membranes were washed again in TBST. Blots were developed using Super Signal Pico Substrate, and images were taken using a Kodak image station. Protein bands were analyzed using the Fluor-s Gel Imaging Analysis System and normalized to $\beta$-actin in the sample.

Analysis of caspase-9 and caspase-3 activation. MG-63 cells were cultured in 6-well plates at a concentration of $2 \times 10^{5}$ cells/ well and treated with various concentrations of quercetin for $48 \mathrm{~h}$. The activities of caspase- 9 and caspase- 3 were determined by a colorimetric assay using the caspase- 9 and caspase- 3 activation kits (Invitrogen) according to the manufacturer's instructions. Briefly, the cells were lysed with provided lysis buffer for $30 \mathrm{~min}$ on ice and centrifuged at $16000 \mathrm{x}$ g for $10 \mathrm{~min}$. The protein concentration was measured by the BCA assay with bovine serum albumin as the standard. Then, $100 \mu \mathrm{g}$ of the protein were incubated with $50 \mu \mathrm{l}$ of the colorimetric tetrapeptides, Leu-Glu-His-Asp (LEHD)-p-nitroaniline (pNA) (specific substrate of caspase-9) or Asp-Glu-Val-Asp (DEAD)-pNA (specific substrate of caspase-3) at $37^{\circ} \mathrm{C}$ for $2 \mathrm{~h}$. The samples were read at $405 \mathrm{~nm}$ in an ELISA reader (BioTek, Model EXL800, USA).

Statistical analysis. Data were analyzed using the SPSS package for Windows (Version 13.0). The quantitative data were expressed as the means \pm standard deviation (SD). Statistical analysis of the data was performed using the Student's t-test
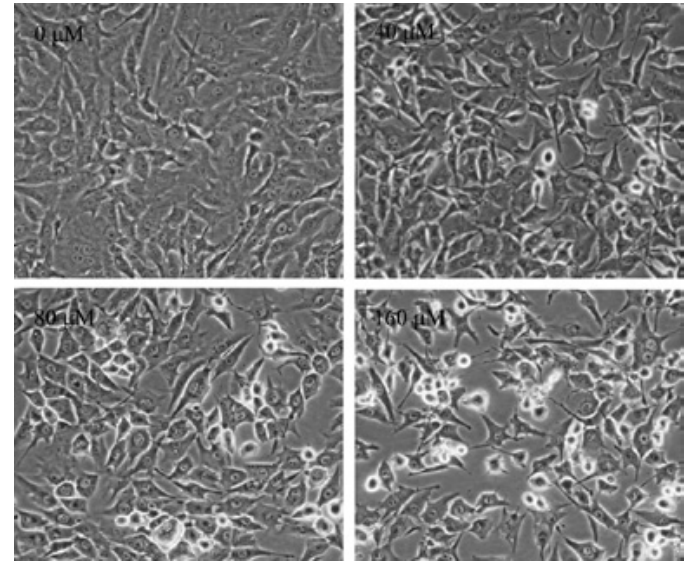

Figure 3. Effect of quercetin on the morphological changes of MG-63 cells (x100). MG-63 cells were treated with the indicated concentrations of quercetin for $48 \mathrm{~h}$ and morphological changes were observed using phasecontrast microscopy.

and ANOVA. Differences with P-values of $<0.05$ were considered statistically significant.

\section{Results}

Quercetin reduces the viability of $M G-63$ cells. The effect of quercetin on the viability of MG-63 cells was determined by MTT assay. As shown in Fig. 2A, after 48 h, the cell viability was reduced to $89.47 \pm 3.63 \%, 77.65 \pm 4.77 \%, 68.14 \pm 5.29 \%$, $51.48 \pm 4.96 \%, 33.93 \pm 4.83 \%$ and $18.27 \pm 4.42 \%$ when cells were treated with quercetin at the final concentrations of $20,40,80$, 160,240 and $320 \mu \mathrm{M}$, respectively, significantly lower than that of the control cells $(98.28 \pm 2.44 \%)(\mathrm{P}<0.01)$, indicating that the inhibitory effects were dose-dependent and the estimated halfmaximal inhibitory concentration (IC50) value was $160 \mu \mathrm{M}$. Hence, $160 \mu \mathrm{M}$ was selected in the following experiments. As shown in Fig. 2B, quercetin reduced cell viability in a timedependent manner.

Quercetin induces morphological changes in MG-63 cells. The effect of quercetin on MG-63 cell morphology was evaluated by phase-contrast microscopy. As shown in Fig. 3, control MG-63 cells appeared as densely disorganized multilayers, whereas many of the cells became shrunken and round-shaped, and detached from each other or floated in the medium after incubation with various concentrations of quercetin for $48 \mathrm{~h}$, suggesting that quercetin can reduce the viability of MG-63 cells.

Quercetin induces apoptosis of MG-63 cells. To further investigate whether the suppressive effect of quercetin on cell viability is due to apoptosis, MG-63 cells were subjected to FACS analysis after Annexin-V/PI staining. In the FACS diagram, LL (Annexin V/PI double-negative population) indicates viable cells; LR or UR (Annexin V-positive/PI-negative or Annexin V/PI double-positive population) represents cells undergoing early or late apoptosis respectively. As shown in Fig. 4A and C, the percentages of cells undergoing apoptosis (including the early and late apoptotic cells) were $21.28 \pm 3.07 \%$, $33.23 \pm 5.78 \%$ and $48.52 \pm 5.33 \%$ when the final concentrations 
A

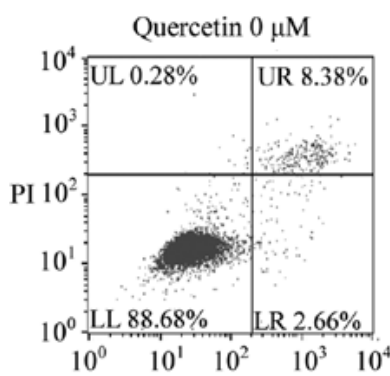

B

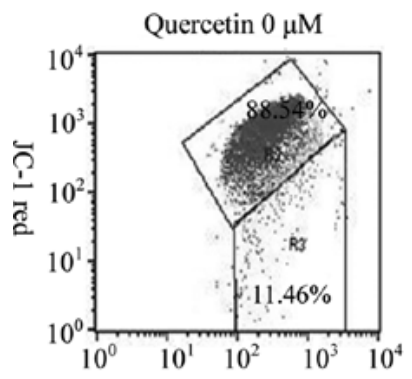

Quercetin $40 \mu \mathrm{M}$

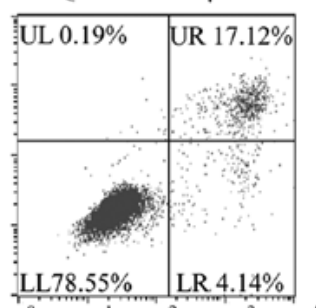

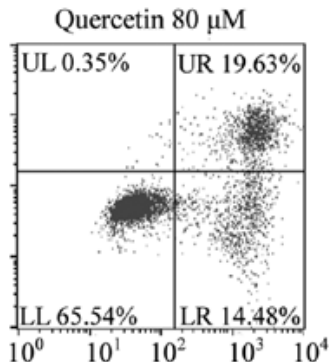

Quercetin $160 \mu \mathrm{M}$

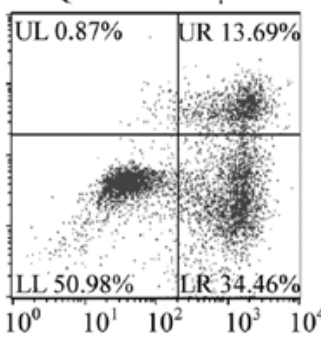

Annexin-V FITC
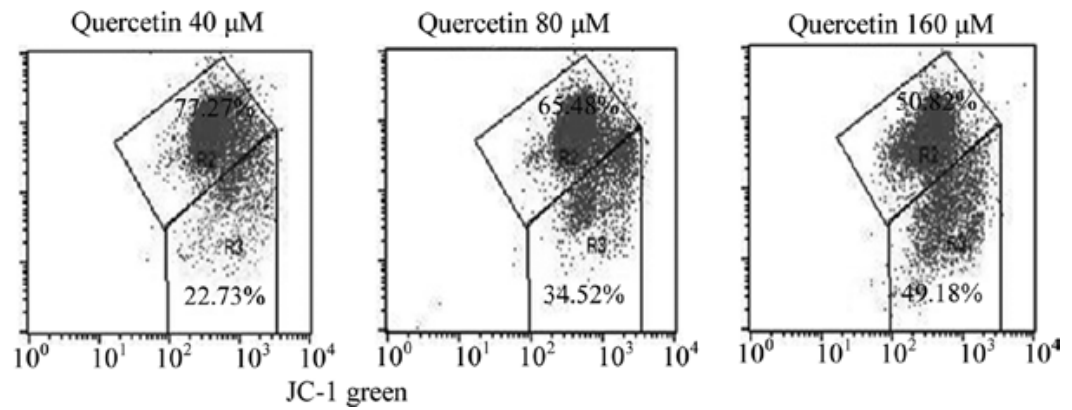

C

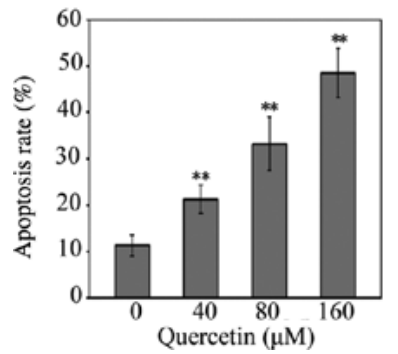

D

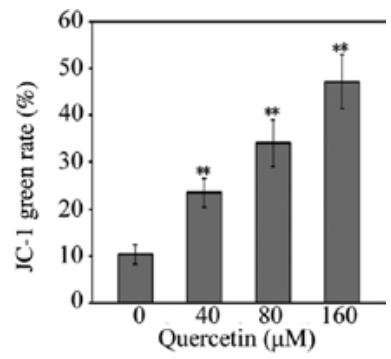

Figure 4. Effect of quercetin on the apoptosis of MG-63 cells. (A) After treatment with the indicated concentrations of quercetin for 48 h, MG-63 cells were collected and stained with Annexin V/PI followed by FACS analysis. Representative FACS analysis scatter-grams of Annexin V/PI staining displays four different cell populations labeled as: LL (lower left), double-negative stained cells indicating live cell population; LR (lower right), Annexin V-positive/PI-negative stained cells representing early apoptosis; UR (upper right), Annexin V/PI double-positive stained cells displaying late apoptosis; UL (upper left), Annexin V-negative and PI-positive stained cells showing dead cells. (B) MG-63 cells were treated with the indicated concentrations of quercetin for 48 h and stained with JC-1. The mean JC-1 fluorescence intensity was detected using FACS analysis. (C) Quantification of apoptosis in MG-63 cells by FACS analysis. The data shown are the averages \pm SD (error bars), ${ }^{* *} \mathrm{P}<0.01$, significant vs. control cells. (D) Quantification of the loss of $\Delta \psi \mathrm{m}$ in MG-63 cells by FACS analysis. The data shown are the averages $\pm \mathrm{SD}$ (error bars), ${ }^{* * *} \mathrm{P}<0.01$, compared to the control cells..

of quercetin were 40,80 and $160 \mu \mathrm{M}$, respectively, significantly higher than the control cells $(11.26 \pm 2.23 \%)(\mathrm{P}<0.01)$. This indicates that quercetin induces MG-63 cell apoptosis in a dose-dependent manner.

Quercetin induces the loss of $\Delta \psi m$. To confirm the pro-apoptotic effect of quercetin, we then investigated the influence of quercetin on the loss of $\Delta \psi \mathrm{m}$, a typical feature of apoptosis. Change in $\Delta \psi \mathrm{m}$ was detected by FACS analysis after JC-1 staining. JC-1 selectively enters the mitochondria and forms J-aggregates with intense red fluorescence $(590 \mathrm{~nm})$ in healthy cells. In apoptotic cells, the altered mitochondrial transmembrane potential causes JC-1 to remain in the cytoplasm in monomeric form, showing green fluorescence $(525 \mathrm{~nm})$. These fluorescence changes can be detected by FACS using JC-1 green and red channels. As shown in Fig. 4B and D, JC-1 fluorescence shifted from a JC-1-green-bright/JC-1-red-bright signal in the control MG-63 cells to a JC-1-green-bright/ JC-1-red-dim signal in the cells treated with quercetin in a dose-dependent fashion. The percentages of JC-1-green-bright cells with $40 \mu \mathrm{M}(23.53 \pm 3.10 \%), 80 \mu \mathrm{M}(34.05 \pm 4.96 \%)$ and
$160 \mu \mathrm{M}(47.18 \pm 5.67 \%)$ of quercetin were significantly higher than the control cells $(10.38 \pm 2.12 \%, \mathrm{P}<0.01)$, indicating the quercetin-induced loss of $\Delta \psi \mathrm{m}$ in MG-63 cells.

Quercetin decreases the expression of anti-apoptotic Bcl-2 and increases pro-apoptotic Bax and cytochrome $C$. Bcl-2 family proteins, including anti-apoptotic members, such as Bcl-2 and pro-apoptotic members, such as Bax and cytochrome C, play important roles in mitochondrial-mediated apoptosis regulation. To further investigate how quercetin induces cancer cell apoptosis, the mRNA transcription and protein expression of Bcl-2, Bax and cytochrome $\mathrm{C}$ in quercetin-treated cells were evaluated by RT-PCR and Western blot analysis, respectively. The results of the RT-PCR assay showed that quercetin treatment profoundly reduced the transcription of the Bcl-2 gene and increased Bax transcription in MG-63 cells compared to the control cells $(\mathrm{P}<0.01)$ (Fig. 5A-C). The results were confirmed by Western blot analysis. In addition, cytochrome $\mathrm{C}$ protein expression was significantly increased by quercetin treatment $(\mathrm{P}<0.01$, vs. control) (Fig. 6A-D). This suggests that quercetin induces mitochondrial-dependent apoptosis in 

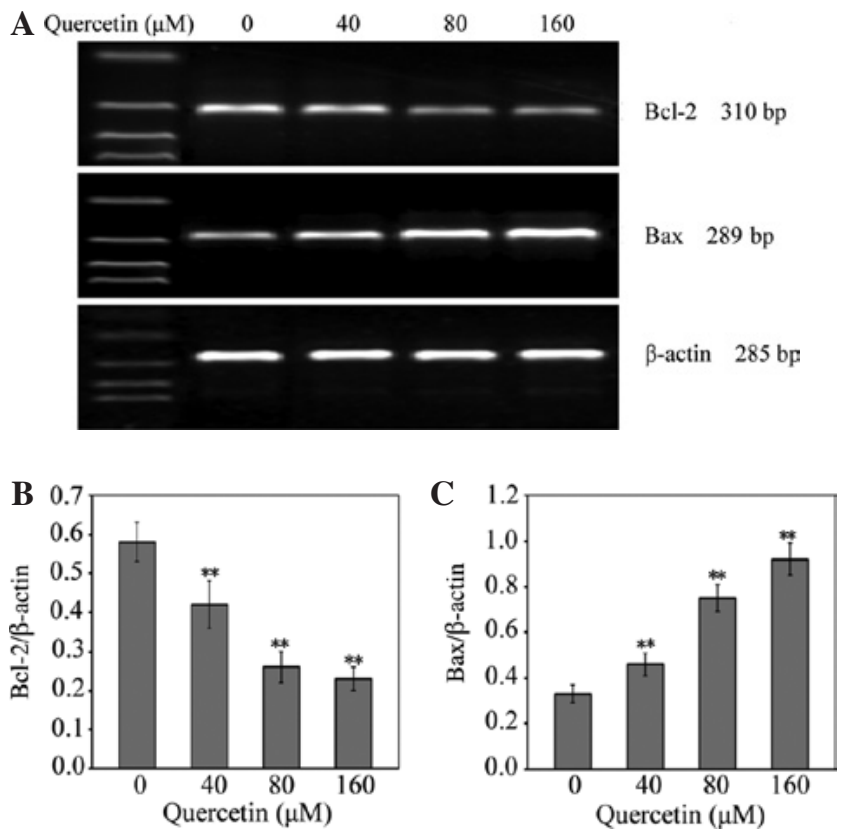

Figure 5. Effect of quercetin on the mRNA expression of Bcl-2 and Bax in MG-63 cells. MG-63 cells were treated with the indicated concentrations of quercetin for $48 \mathrm{~h}$. (A) The mRNA expression of Bcl-2 and Bax in quercetintreated and control cells was determined by RT-PCR. $\beta$-actin was used as the internal control for the RT-PCR assays. Quantification of RT-PCR analysis, the data shown are the averages $\pm \mathrm{SD}$ (error bars), ${ }^{* *} \mathrm{P}<0.01$, compared to the control cells. (B) The mRNA expression of Bcl-2 in quercetin-treated and control cells. (C) The mRNA expression of Bax in quercetin-treated and control cells.

MG-63 cells via regulating the expression of the Bcl-2 family proteins.

Quercetin treatment results in increased activities of caspase-9 and caspase-3. The mitochondrial membrane permeabilization results in the release of numerous apoptogenic proteins from the mitochondria, triggering the activation of caspase- 9 and caspase-3, and eventually leading to apoptosis. To investigate the downstream effectors in the apoptotic signaling pathway, the activation of caspase- 9 and caspase- 3 was detected by a colorimetric assay using the specific chromophores, LEHD-pNA (specific substrate of caspase-9) and DEVD-pNA (specific substrate of caspase-3), respectively. As shown in Fig. 7A and $\mathrm{B}$, quercetin treatment significantly and dose-dependently increased the activities of caspase- 9 and caspase- 3 in MG-63 cells compared to the control cells $(\mathrm{P}<0.05$ and $\mathrm{P}<0.01$, respectively). Taken together, these results suggest that quercetin promotes MG-63 cell apoptosis via the mitochondrial-dependent pathway.

\section{Discussion}

Our study demonstrates that quercetin induces the loss of $\Delta \psi \mathrm{m}$, upregulates the expression of the proapoptotic proteins, Bax and cytochrome $\mathrm{C}$, and downregulates the expression of the antiapoptotic protein, Bcl-2, resulting in the increased activity of caspase- 9 and caspase- 3 . Hence, quercetin reduces the viability of MG-63 cell at least via the mitochondrialdependent pathway.
A

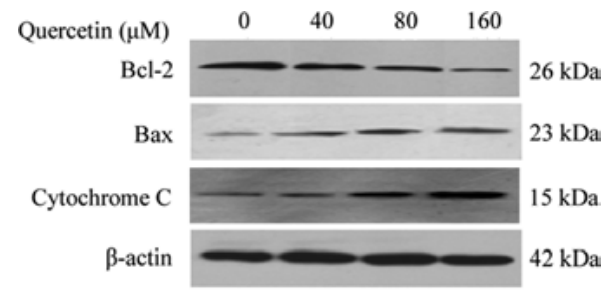

B

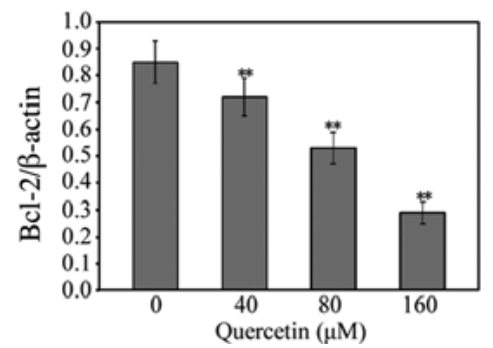

C

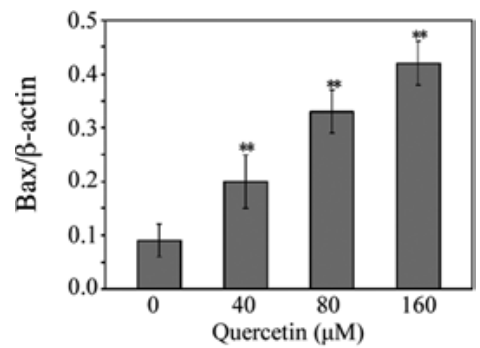

D

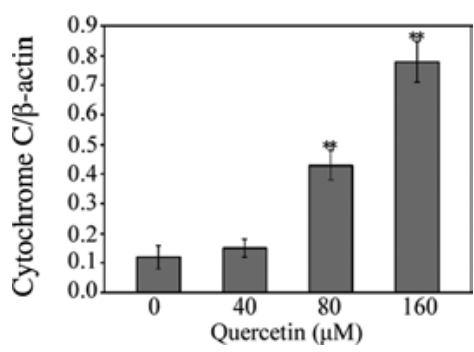

Figure 6. Effect of quercetin on the protein expression levels of Bcl-2, Bax and cytochrome C in MG-63 cells. (A) The protein expression levels of Bcl-2, Bax and cytochrome $\mathrm{C}$ were analyzed by Western blot analysis. $\beta$-actin was used as the internal control for the Western blot analysis. Quantification of Western blot analysis. The data shown are the averages \pm SD (error bars), ${ }^{* *} \mathrm{P}<0.01$, significant vs. control cells. (B) The protein expression levels of Bcl-2 in quercetin-treated and control cells. (C) The protein expression levels of Bax in quercetin-treated and control cells. (D) The protein expression levels of cytochrome $\mathrm{C}$ in quercetin-treated and control cells.

Tumor cells are characterized by an unregulated increase in cell proliferation and/or a reduction in cell apoptosis. Moreover, disrupted apoptosis contributes to drug resistance of tumor cells, which has become a significant obstacle for the successful management of patients with malignant tumors, including OS (18). The growing amount of evidence from studies on tumor cell cultures and animal tumor models demonstrates that a large number of natural products from the diet can sensitize tumor cells to anti-cancer therapies $(19,20)$. For cancer prevention and chemotherapy, plant-derived natural products are an invaluable treasure and worthy to be further explored.

Quercetin, one of the major dietary flavonoids found in various teas, vegetables and fruits, has been reported to exert a broad range of anticancer effects. It can selectively inhibit the proliferation of tumor cells and induce cell apoptosis $(21,22)$. 
A

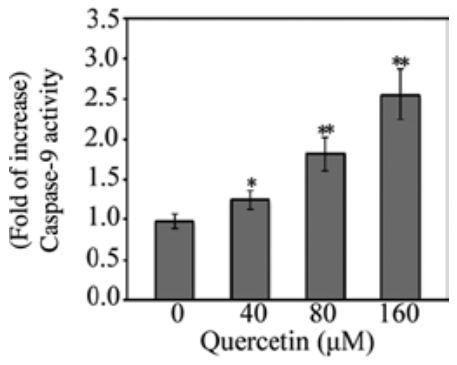

B

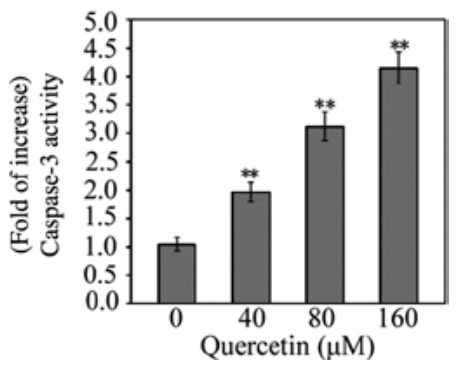

Figure 7. Effect of quercetin on the activity of caspases in MG-63 cells. MG-63 cells were treated with the indicated concentrations of quercetin for $48 \mathrm{~h}$. Caspase- 9 and caspase- 3 activities were determined by a colorimetric assay. The data were normalized to the caspase activities of the control cells and represented as the fold of the control. The data shown are the averages $\pm \mathrm{SD}$ (error bars). ${ }^{*} \mathrm{P}<0.05,{ }^{* *} \mathrm{P}<0.01$, compared to the control cells. (A) The activity of caspase-9 in quercetin-treated and control MG-63 cells. (B) The activity of caspase- 3 in quercetin-treated and control MG-63 cells.

Suh et al reported that the antiproliferative action of quercetin human osteosarcoma cells appeared to be linked to apoptotic cell death based on increase in the sub-G(1) apoptotic cell population (9). However, the underlying molecular mechanism is not fully understood.

Apoptosis is triggered by two different signals. The extrinsic apoptotic pathway involves death receptors, which respond mainly to extracellular stimuli. The intrinsic apoptotic pathway involves the mitochondria that are activated by modulators within the cell itself $(23,24)$. Both pathways eventually lead to the activation of caspases and nucleases, resulting in cell death. The mitochondria continuously collect information on various aspects of signal transduction cascades and cellular metabolism, process this information, then decide on the fate of cells and participate in the regulation of cell death. MOMP is a key commitment step in the induction of cellular apoptosis, as it is often required for the activation of the caspase proteases that cause apoptotic cell death (25). During the process of MOMP, the electrochemical gradient across the mitochondrial membrane collapses, hence the loss of $\Delta \psi \mathrm{m}$ is a hallmark for apoptosis. Our data clearly show that treatment with quercetin leada to the collapse of $\Delta \psi \mathrm{m}$ in MG-63 cells.

Mitochondrial-dependent apoptosis upstream of caspase activation is regulated by members of the Bcl-2 family (26). Apoptosis-associated MOMP is known to require pro-apoptotic Bax-like proteins, in the regulation of the formation of pores in the mitochondria. Anti-apoptotic Bcl-2-like proteins in mitochondrial morphogenesis are functionally distinct from their role in apoptosis. Therefore, the ratio of Bax to Bcl-2 is critical for determining the release of many apoptogenic proteins from the mitochondrial intermembrane space, such as cytochrome C $(27,28)$. Cytochrome C can activate caspase-9.
The activated caspase- 9 cleaves and activates executioner caspases, such as caspase-3, then drives the caspase cascade and the cell death mechanism (29).

In this study, we demonstrate that quercetin dose-dependently upregulates Bax expression and downregulates Bcl-2 expression in MG-63 cells. This indicates that quercetin induces apoptosis by affecting the ratio of $\mathrm{Bax} / \mathrm{Bcl}-2$. This results in enhanced cytochrome $\mathrm{C}$ expression and increases the activities of caspase- 9 and caspase- 3 .

In conclusion, our data demonstrate that quercetin induces MG-63 cell apoptosis via the mitochondrial-dependent pathway. These results indicate that quercetin could be a potential novel therapeutic agent for the treatment of OS. Further studies are required in order to find out whether quercetin can synergize with other chemotherapy drugs.

\section{Acknowledgements}

This study was supported by the National Natural Science Foundation of China (Grant no. 30772697).

\section{References}

1. Rippo MR, Villanova F, Tomassoni Ardori F, et al: Dexamethasone affects Fas- and serum deprivation-induced cell death of human osteoblastic cells through survivin regulation. Int J Immunopathol Pharmacol 23: 1153-1165, 2010.

2. Oertel S, Blattmann C, Rieken S, et al: Radiotherapy in the treatment of primary osteosarcoma-a single center experience. Tumori 96: 582-588, 2010.

3. Won KY, Lee CH, Kim YW and Park YK: Primary giantcell-rich osteosarcoma of the urinary bladder: usefulness of osteocalcin and osteonectin immunohistochemical staining and literature review. Pathology 43: 161-164, 2011.

4. Gordon EM and Hall FL: Noteworthy clinical case studies in cancer gene therapy: tumor-targeted Rexin-G advances as an efficacious anti-cancer agent. Int J Oncol 36: 1341-1353, 2010.

5. Chen C, Zhou J and Ji C: Quercetin: a potential drug to reverse multidrug resistance. Life Sci 87: 333-338, 2010.

6. Murakami A, Ashida $\mathrm{H}$ and Terao J: Multitargeted cancer prevention by quercetin. Cancer Lett 269: 315-325, 2008.

7. Galluzzo P, Martini C, Bulzomi P, et al: Quercetin-induced apoptotic cascade in cancer cells: antioxidant versus estrogen receptor alpha-dependent mechanisms. Mol Nutr Food Res 53: 699-708, 2009.

8. Chang YF, Hsu YC, Hung HF, et al: Quercetin induces oxidative stress and potentiates the apoptotic action of 2-methoxyestradiol in human hepatoma cells. Nutr Cancer 61: 735-745, 2009.

9. Suh DK, Lee EJ, Kim HC and Kim JH: Induction of G(1)/S phase arrest and apoptosis by quercetin in human osteosarcoma cells. Arch Pharm Res 33: 781-785, 2010.

10. Ferraresi R, Troiano L, Pinti M, et al: Resistance of mtDNAdepleted cells to apoptosis. Cytometry A 73: 528-537, 2008.

11. Wakshlag JJ and Balkman CE: Effects of lycopene on proliferation and death of canine osteosarcoma cells. Am J Vet Res 71: 1362-1370, 2010.

12. Landriscina M, Laudiero G, Maddalena F, et al: Mitochondrial chaperone Trapl and the calcium binding protein Sorcin interact and protect cells against apoptosis induced by antiblastic agents. Cancer Res 70: 6577-6586, 2010.

13. Cao X, Bennett RL and May WS: c-Myc and caspase-2 are involved in activating Bax during cytotoxic drug-induced apoptosis. J Biol Chem 283: 14490-14496, 2008.

14. Manfredi G, Kwong JQ, Oca-Cossio JA, et al: BCL-2 improves oxidative phosphorylation and modulates adenine nucleotide translocation in mitochondria of cells harboring mutant mtDNA. J Biol Chem 278: 5639-5645, 2003.

15. Soriano ME and Scorrano L: The interplay between BCL-2 family proteins and mitochondrial morphology in the regulation of apoptosis. Adv Exp Med Biol 687: 97-114, 2010.

16. Ryu SY, Peixoto PM, Teijido O, Dejean LM and Kinnally KW: Role of mitochondrial ion channels in cell death. Biofactors 36 : 255-263, 2010. 
17. Autret A and Martin SJ: Emerging role for members of the Bcl-2 family in mitochondrial morphogenesis. Mol Cell 36: 355-363, 2009.

18. Broadhead ML, Clark JC, Dass CR, Choong PF and Myers DE: Therapeutic targeting of osteoclast function and pathways Expert Opin Ther Targets 15: 169-181, 2011.

19. Colomer R and Menéndez JA: Mediterranean diet, olive oil and cancer. Clin Transl Oncol 8: 15-21, 2006.

20. Roomi MW, Roomi N, Ivanov V, Kalinovsky T, Niedzwiecki A and Rath M: Inhibitory effect of a mixture containing ascorbic acid, lysine, proline and green tea extract on critical parameters in angiogenesis. Oncol Rep 14: 807-815, 2005.

21. Zanini C, Giribaldi G, Mandili G, et al: Inhibition of heat shock proteins (HSP) expression by quercetin and differential doxorubicin sensitization in neuroblastoma and Ewing's sarcoma cell lines. J Neurochem 103: 1344-1354, 2007.

22. Vargas AJ and Burd R: Hormesis and synergy: pathways and mechanisms of quercetin in cancer prevention and management. Nutr Rev 68: 418-428, 2010.

23. Park H, Bergeron E, Senta $\mathrm{H}$, et al: Sanguinarine induces apoptosis of human osteosarcoma cells through the extrinsic and intrinsic pathways. Biochem Biophys Res Commun 399: 446-451, 2010
24. Gazitt Y, Kolaparthi V, Moncada K, Thomas C and Freeman J: Targeted therapy of human osteosarcoma with 17AAG or rapamycin: characterization of induced apoptosis and inhibition of mTOR and Akt/MAPK/Wnt pathways. Int J Oncol 34: 551-561, 2009.

25. Gagiannis S, Müller M, Uhlemann S, et al: Parathyroid hormonerelated protein confers chemoresistance by blocking apoptosis signaling via death receptors and mitochondria. Int J Cancer 125: 1551-1557, 2009.

26. Leibowitz B and Yu J: Mitochondrial signaling in cell death via the Bcl-2 family. Cancer Biol Ther 9: 417-422, 2010.

27. Kaseta MK, Khaldi L, Gomatos IP, et al: Prognostic value of bax, bcl-2, and p53 staining in primary osteosarcoma. J Surg Oncol 97: 259-266, 2008

28. Eliseev RA, Dong YF, Sampson E, et al: Runx2-mediated activation of the Bax gene increases osteosarcoma cell sensitivity to apoptosis. Oncogene 27: 3605-3614, 2008.

29. Lim ML, Chen B, Beart PM and Nagley P: Relative timing of redistribution of cytochrome $\mathrm{c}$ and Smac/DIABLO from mitochondria during apoptosis assessed by double immunocytochemistry on mammalian cells. Exp Cell Res 312: 1174-1184, 2006. 